\title{
A series of helical $\alpha$-synuclein fibril polymorphs are populated in the presence of lipid vesicles
}

\author{
Richard M. Meade ${ }^{1}$, Robert J. Williams $\mathbb{D}^{1}$ and Jody M. Mason (D) $^{1 凶}$
}

a-Synuclein (aS) deposition is a defining characteristic of Parkinson's disease (PD) pathology, and other synucleinopathies. aS aggregates in disease, leading to the generation of neuronal inclusions known as Lewy bodies. These accumulate in the cytoplasmic space of dopaminergic neurons in the substantia nigra pars compacta region of the brain, causing cell death, resulting in decreased dopamine levels, and ultimately PD symptoms. To date, a significant proportion of structural information has arisen from in vitro studies using recombinantly purified forms of the protein, often failing to acknowledge that aS is natively located in the presence of phospholipids, where it likely plays a direct role in regulating synaptic vesicle function and neurotransmission. Here we present a series of macromolecular aS assemblies not previously described that form in the presence of lipid vesicles. These fibrillar structures are striking in both their large size relative to those previously reported and by their varying helical content, from ribbons to wave-like helices of long pitch shortening to those more compact and bulkier. These studies provide the foundation for more detailed structural analysis, and may offer new possibilities to further define disease-relevant versions of the protein that are accessible to pharmacological intervention.

npj Parkinson's Disease (2020)6:17; https://doi.org/10.1038/s41531-020-00122-1

\section{INTRODUCTION}

a-Synuclein (aS), which has been described as intrinsically disordered or helical, is capable, under certain conditions, of aggregating into a range of different amyloid fibril morphologies ${ }^{1}$. These structures are typically probed via imaging techniques that include electron microscopy (EM) and atomic force microscopy, and more recently at the atomic level using solid-state nuclear magnetic resonance ${ }^{2}$, and increasingly, $\mathrm{CryoEM}^{3-8}$. Many approaches describe structures gained via recombinant means under in vitro conditions $s^{2-5,7,9,10}$. Others have employed the use of lipids during in vitro experiments, since this is critical to aS function in situ ${ }^{6,11-17}$. More recent studies are emerging in which aggregates of aS from specific neurodegenerative diseases and key regions of the brain are isolated in small quantities ${ }^{8,18}$. The latter have then been used to seed recombinant forms of aS produced in bacteria or cell culture, with the assumption that seed conformation is maintained in mature fibres, without polymorphic shift, and thus those observed within imaging is of the diseaserelevant type. To date, all but one of the structures are defined by a double protofilament. Most, but not all, display rotational symmetry about the axis of the fibril.

In particular, the bacterial approach has been used to describe an increasing number of fibril morphologies. Using this approach the first morphologies were described-type $1 \mathrm{a}\left({ }^{\prime}\right.$ rods $^{3,5}$ ) and $1 \mathrm{~b}$ $\left(\right.$ 'twisters $\left.{ }^{\prime 5}\right)$, as well as types $2 a$ and $2 b^{9}$. This approach has also extended to structures of early-onset as mutants, including $\mathrm{E}_{4} 6 \mathrm{~K}^{5,19}$ and $\mathrm{H} 50 \mathrm{Q}^{19}$. Studying aS using seeds isolated from brain samples and growing mature fibres from them using additional protein produced by bacterial/mammalian cells is also beginning to yield new structures ${ }^{18}$. For example, in multiple system atrophy (MSA) two further unique morphologies (named Type I and Type II) have been described; these contain non-proteinaceous, potentially negatively charged molecules, at the protofilament interface. Interestingly, in both structures each protofibril within the mature fibril adopts a different morphology, rendering the fibrils asymmetric ${ }^{8}$. In contrast to MSA-derived filaments, those seeded from aS isolated from individuals with dementia with Lewy bodies were reported to lack any twist and were reported to be thinner than those from the brains of individuals with MSA, suggested another currently uncharacterised morphological fibril type.

All structures identified to date display a mature fibril width of $\sim 10 \mathrm{~nm}$ and a typical helical pitch in the Angstrom range (e.g. $\sim 460 \AA$ for twisters, or $\sim 920 \AA$ for rods). Here we describe very different and much larger assemblies that have not been previously reported, and which form specifically in the presence of lipid vesicles. This is particularly interesting given the emerging discussion over whether Parkinson's disease (PD) is a proteinopathy or lipidopathy ${ }^{20}$. These newly reported structures are able to be readily viewed using transmission EM (TEM) and range in polymorph from structures that are ribbon like to those that are highly helical and compact, with a range of observed helical assemblies in between. Interestingly, the addition of lipid vesicles to the monomeric as sample appears to be an absolute requirement for the morphological shift to such larger assemblies. We believe, given the role that aS plays within the cell, that these structures could have biological or pathological relevance and therefore far-reaching implications in defining a disease-relevant conformation towards identification of a druggable target.

\section{RESULTS AND DISCUSSION}

Lipid vesicle-induced fibril polymorphs

The addition of lipids to aS has been reported to modulate misfolding and toxicity ${ }^{21}$, causing the protein to form an extended helical structure on small unilamellar vesicles (SUVs). The binding of aS to lipid membranes has complex effects on the latter ${ }^{13}$, altering the curvature of the bilayer structure and leading to the formation of small vesicles ${ }^{14}$. as has been shown to be capable of

\footnotetext{
${ }^{1}$ Department of Biology and Biochemistry, University of Bath, Claverton Down, Bath BA2 7AY, UK. ${ }^{凶}$ email: j.mason@bath.ac.uk
} 


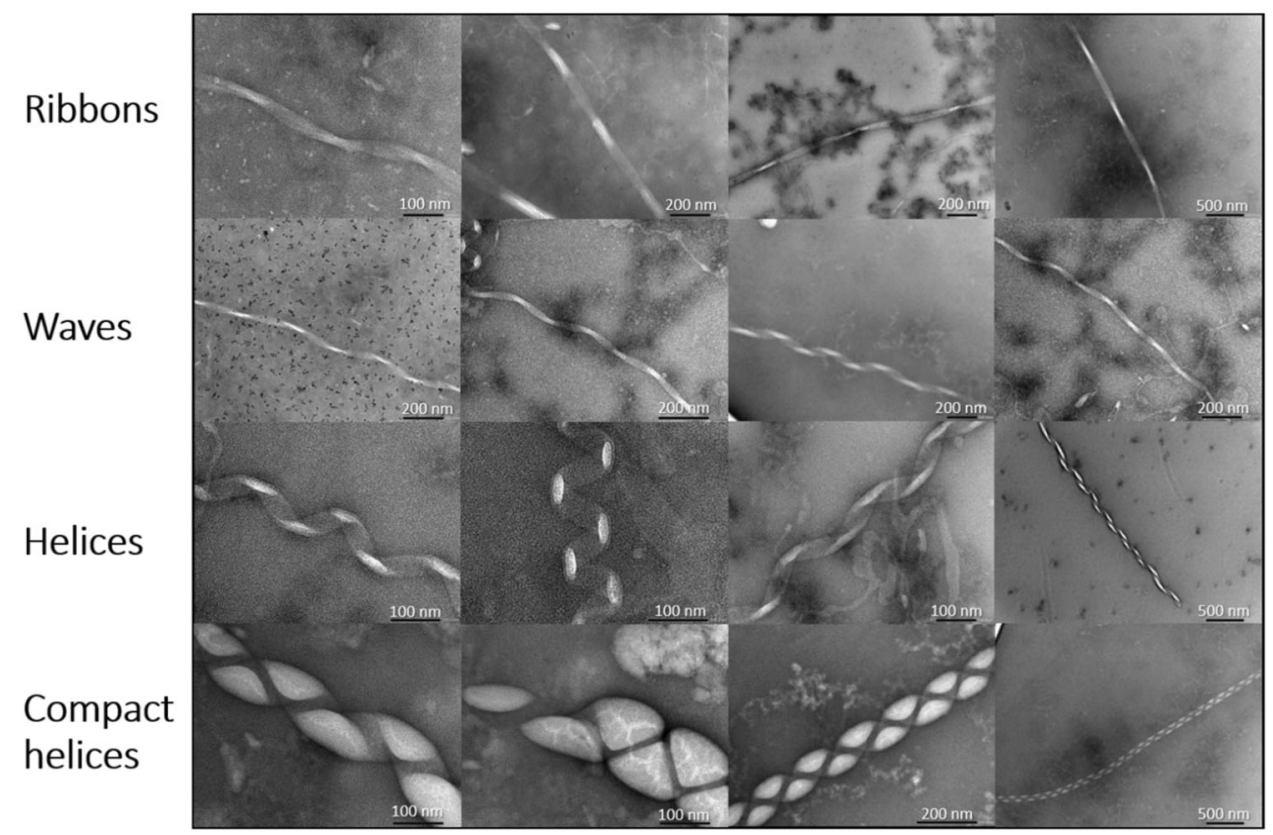

Fig. 1 A variety of large fibril polymorphs are formed upon incubation of aS with DMPS lipid vesicles. These structures were formed by aggregating $100 \mu \mathrm{M} \alpha \mathrm{S}$ in the presence of $200 \mu \mathrm{M}$ DMPS lipid vesicles at $30^{\circ} \mathrm{C}$ for $190 \mathrm{~h}$. The structures have initially been divided into four subtypes with varying degrees of helicity named ribbons, waves, helices and compact helices.

bending membranes consisting of negatively charged phospholipid vesicles and forming tubules from large lipid vesicles ${ }^{22}$.

Using TEM we report for the first time the formation of a range of large macromolecular structures that vary widely in their morphology (Fig. 1). In particular, we describe the formation of a much larger type of macro-assembly that can form in the presence of lipid vesicles. Specifically, 1,2-dimyristoyl-sn-glycero3-phospho-L-serine (DMPS) was used to create SUVs, at neutral pH $\left(\mathrm{pH} 6.5,30^{\circ} \mathrm{C}\right)$, as previously described ${ }^{15,23,24}$. DMPS vesicles were chosen as a model phospholipid system since they are a key component within dopaminergic synaptic vesicles, and the negative charge on their surface has been shown to be capable of significantly promoting aS primary nucleation ${ }^{15,16}$. Interestingly, DMPS lipid vesicles have been previously shown to act by forming kinetically trapped aS protofibrils ${ }^{25}$. Moreover, lipid-induced fibril production has been shown to be strongly affected by early-onset mutations, which can induce dramatic changes in such crucial processes thought to be associated with the initiation and spreading of aS aggregation ${ }^{26}$. Phospholipid biosynthetic enzymes have also been seen to be elevated in the substantia nigra of PD patients ${ }^{27}$. Specific membrane interactions can therefore play a key role in triggering a conversion of aS from a soluble to aggregated form that is associated with disease.

To induce the formation of the macromolecular structures, briefly, $100 \mu \mathrm{M}$ monomeric aS (Supplementary Fig. 1) was mixed with $200 \mu$ M DMPS SUVs, of $\sim 30-40 \mathrm{~nm}$ diameter (as measured by dynamic light scattering (DLS); Supplementary Fig. 2), under quiescent conditions (Fig. 2). The solution was left to aggregate, as monitored by ThT fluorescence, for a period of $48 \mathrm{~h}$, allowing the fluorescence to progress through the lag and exponential phases of aggregation before plateauing at this time at the stationary phase. A sample was collected from this phase and analysed by TEM (Fig. 2a). During this time distinctive fibril-like structures were observed to emerge from the surface of the SUVs that are similar to those reported by others in the presence of $\mathrm{DMPS}^{6,15}$. In particular, these meandering fibril-like structures, were $\sim 5 \mathrm{~nm}$ in diameter, suggesting that they are protofibrils of the singlestranded variety ${ }^{2}$. Also observed were wider fibrillar structures, measuring $10 \mathrm{~nm}$ in diameter (Supplementary Fig. 3), suggestive

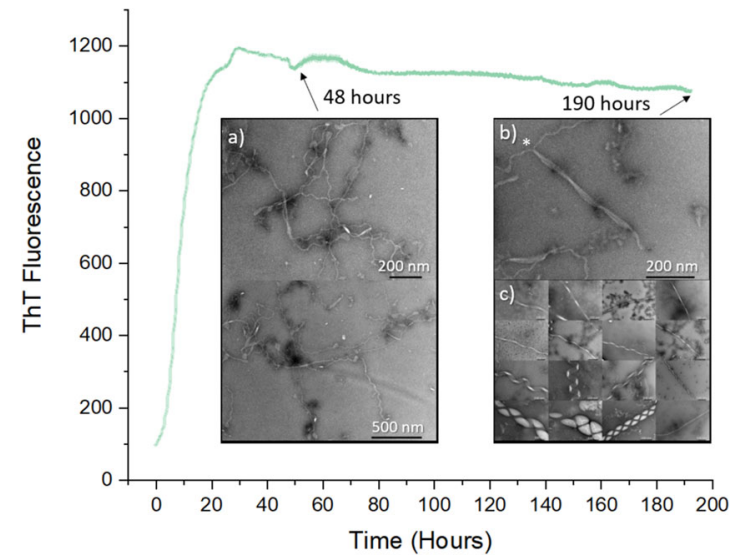

Fig. 2 Lipid-induced aS aggregation followed by ThT fluorescence. Aggregation of $100 \mu \mathrm{M} \alpha \mathrm{S}$ in the presence of $200 \mu \mathrm{M}$ DMPS lipid vesicles at $30^{\circ} \mathrm{C}$, followed by ThT fluorescence and TEM. a TEM samples taken at $48 \mathrm{~h}$ show the formation of meandering fibril-like structures growing from the surface of punctate lipid vesicles. These are observed to be $\sim 5 \mathrm{~nm}$ in width and straight, or $\sim 10 \mathrm{~nm}$ in width (Supplementary Fig. 3). b TEM samples taken at $\approx 190 \mathrm{~h}$ show two meandering $10 \mathrm{~nm}$ fibrils fusing to form a 40-nm-wide ribbon-like structure* $^{*}$. C TEM samples observed at $\approx 190 \mathrm{~h}$ display a range of macromolecular polymorphs (image is shown in more detail in Fig. 1).

of a more mature double strand fibril structure that is consistent with previously reported dimensions $s^{3-5}$. The samples were next left to further incubate at $30^{\circ} \mathrm{C}$ until $190 \mathrm{~h}$ had passed, upon which the helical assemblies reported were observed. Since lipidinduced meandering protofibrils may represent kinetically trapped intermediates ${ }^{6,25}$, extended incubation times may be required for conversion to the much larger helical assemblies reported here. Of note during this period is the fact that no detectable change in ThT fluorescence was observed (Fig. 2; Supplementary Fig. 4). This may explain why these structures have not been previously reported, since it also suggests that no additional ThT becomes 
a)

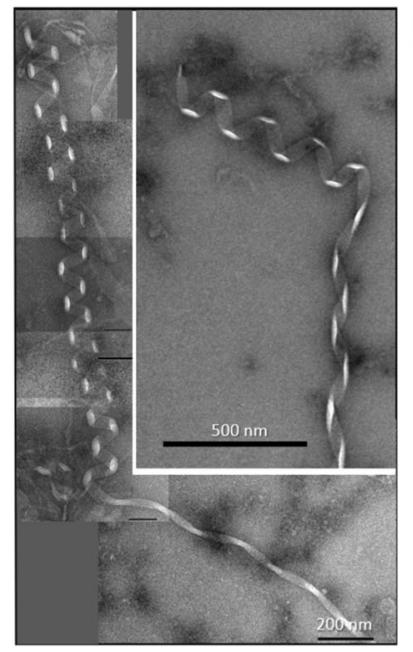

b)

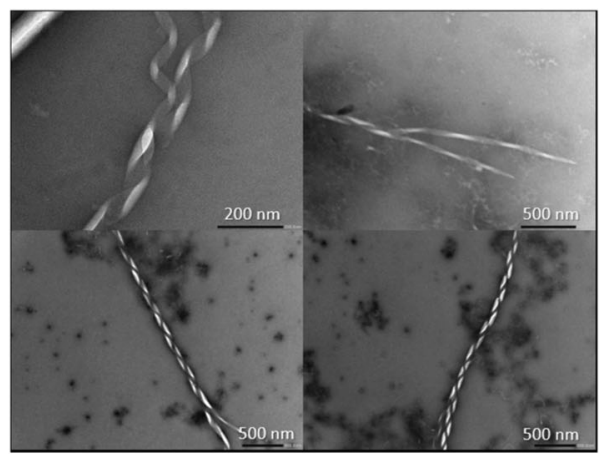

Fig. 3 Polymorph unwinding. a $\alpha$ S polymorph 'helices' are observed to unwind into 'ribbons' and 'waves'. b The $\alpha S$ macromolecular assemblies have been observed to have frayed ends, suggesting a common building block between the different polymorphs.

bound during the formation of these structures. At $190 \mathrm{~h}$ the sample was again analysed by TEM, with fibril-like structures appearing to aggregate into much larger macromolecular assemblies (Fig. 2b). Within the sample a range of macromolecular polymorphs not previously described can be seen ranging in width and helicity (Figs. 1, 2b).

The width of the assemblies are up to ten times larger than aS structures reported previously; up to $100 \mathrm{~nm}$, with helical pitches of $\sim 40-100 \mathrm{~nm}$ (Fig. 1). Observed helicity is also wide ranging; in the most elongated assemblies (Fig. 1), we observe ribbon-like structures ('ribbons') with a pitch $\sim 200 \mathrm{~nm}$, increasing to low helicity structures ('waves') with a similar pitch of $\sim 200 \mathrm{~nm}$. Next, we observe structures that resemble a classic helical signature ('helices') with a pitch of $\sim 100 \mathrm{~nm}$. Finally, very electron-dense compact helical structures ('compact helices') are formed. 'Ribbons', 'waves' and 'helices' are all $\sim 40-50 \mathrm{~nm}$ wide, but as would be expected, 'compact helices' by contrast are much larger at $\sim 100 \mathrm{~nm}$ in width. All structures are capable of reaching many microns in length with some variations in width and helicity. Interestingly, we also observe changes in helical type within the same fibril, suggesting that 'helices', 'waves' and 'ribbons' are interconvertible (Fig. 3a). Finally, we also report upon branched fibril termini, which may represent the formation of the larger assemblies from smaller structures (Fig. 3b). This also suggests that these macromolecular assemblies may be formed by the same common as fibril building blocks that then cluster adjacently along the vertical axis. Reassuringly, the same structures were observed upon three separate repetitions of the experiment. These were undertaken weeks apart and in each case were prepared using both fresh aS and DMPS vesicle preparations (Supplementary Fig. 5), demonstrating both the reproducibility of our findings and similar morphologies of the aS aggregates observed (Supplementary Fig. 6).

Regarding molecular determinants of aS-lipid interaction that may predetermine the formation of the structures we report, it has been proposed that lysine-rich positions within aS may interact with negatively charged lipid head groups, while hydrophobic regions may interact with membrane lipids, leading to an initial helical conformation ${ }^{1,28,29}$. Specifically, the central region of the protein (residues 26-97) has been shown to bind to lipid membranes and positive charge within the $\mathrm{N}$-terminal region may also play a key role in mediating binding to anionic lipids ${ }^{30,31}$. A high local concentration of as at the membrane could potentially lead to primary nucleation and a conversion to $\beta$-sheet formation. The position of single amino acid changes associated with early-onset PD mutations is likely to alter sidechain-sidechain or sidechain-lipid interactions that modulate helix formation, thereby accelerating the pathway toward amyloidosis ${ }^{26}$.

Given the proposed native role of aS in synaptic transmission and signalling, and the clear interaction of aS with dopaminergic neuronal membranes, the discovery of these structures in the presence of phospholipid vesicles is likely to have far-reaching implications towards defining a pathogenic aS conformation that has significant potential to open up new routes towards new conformations and drug targets for diseases in which as aggregates present.

\section{METHODS}

Protein expression and purification of human wild-type aS Wild-type (wt) human aS was recombinantly expressed and purified based on, and modified from, a previously published method (Supplementary Fig. 1) $)^{32,33}$. Briefly, the pET21a plasmid containing the human wt aS (1-140), purchased from Addgene (deposited by the Michal J. Fox Foundation (MJFF), Plasmid \#51486), was transformed into Escherichia coli expression cell line BL21 (DE3). 2xYT overnight cultures containing ampicillin of this human wt aS (1-140) pET21a BL21 (DE3) E. coli strain were used to inoculate 1 I 2xYT cultures, containing $100 \mathrm{mg} \mathrm{I}^{-1}$ ampicillin, and grown at $37^{\circ} \mathrm{C}, 200$ r.p.m. shaking, to $\mathrm{OD}_{600}=0.6-0.8$ and induced with $1 \mathrm{mM}$ isopropyl-1-thio-D-galactopyranoside at $37^{\circ} \mathrm{C}, 200$ r.p.m. shaking, for $4 \mathrm{~h}$ in an Innova 44 Incubator shaker (New Brunswick Scientific). The bacteria were harvested by centrifugation at $4600 \times g$, and resuspended in $40 \mathrm{ml}$ of $20 \mathrm{mM}$ Tris buffer $\mathrm{pH} 8$ containing 1 complete protease inhibitor tablet (Roche) and freeze-thawed at $-20^{\circ} \mathrm{C}$ before lysis, by sonication. The cell debris was discarded by centrifugation at $48,400 \times g$, and the supernatant was collected and boiled at $95^{\circ} \mathrm{C}$ for $10 \mathrm{~min}$. The precipitated protein was removed by centrifugation at $18,500 \times g$. The supernatant was collected, and ammonium sulfate was added to $30 \%$ saturation $\left(0.176 \mathrm{~g} \mathrm{ml}^{-1}\right)$, and left shaking at $20^{\circ} \mathrm{C}$ for $1 \mathrm{~h}$. The precipitated protein, containing the aS, was harvested by centrifugation at $18,500 \times g$, and then resuspended in $50 \mathrm{ml}$ of $20 \mathrm{mM}$ Tris buffer $\mathrm{pH} 8$ by gentle agitation at $4^{\circ} \mathrm{C}$. The proteins were purified by anion exchange chromatography on an AKTA pure purification system (GE Healthcare) with a $5 \mathrm{ml} \mathrm{HiTrap} \mathrm{Q} \mathrm{HP} \mathrm{(GE} \mathrm{Healthcare)} \mathrm{pre-packed} \mathrm{column,} \mathrm{to} \mathrm{remove}$ protein impurities and protein-bound nucleic acids. The purified fractions were combined and further purified by size-exclusion chromatography (SEC), using a HiLoad 16/60 Superdex $75 \mathrm{pg}$ (GE Healthcare) pre-packed purification column, to buffer exchange the aS into the relevant reaction buffer ( $20 \mathrm{mM}$ sodium phosphate buffer $\mathrm{pH} 6.5 / 20 \mathrm{mM}$ sodium acetate $\mathrm{pH}$ 5.0) and ensure that only monomers were collected. Pure monomeric aS was eluted between 54 and $60 \mathrm{ml}$.

The concentration of the purified aS was determined in a 2-mm path length quarts cuvette, using an extinction coefficient of $4836 \mathrm{M}^{-1} \mathrm{~cm}^{-1}$ at 
$280 \mathrm{~nm}$, separated into $1 \mathrm{ml}$ aliquots, snap frozen in liquid $\mathrm{N}_{2}$, and stored at $-80^{\circ} \mathrm{C}$ until required.

The purity of aS following SEC was confirmed by sodium dodecyl sulfate-polyacrylamide gel electrophoresis, and the correct mass was confirmed by mass spectrometry on a Dionex Acclaim RSLC Polar Advantage II (PA2), $2.2 \mu \mathrm{m}, 120 \AA \AA, 2.1 \times 50 \mathrm{~mm}^{2}$ (Thermo Fisher Scientific, California, USA). The deconvoluted average mass of the protein was confirmed as $14,459.749 \mathrm{~m} / z$, representing the mass of wt human aS (1-140). A circular dichroism spectra scan was performed, to confirm the random coil conformation of the monomeric as stock (Supplementary Fig. 5).

\section{Microplate ThT kinetic assays without shaking to measure lipid-induced aggregation}

ThT kinetic assays for lipid induced primary nucleation of aS were performed in a CLARIOstar fluorescence microplate reader (BMG Labtech), under quiescent conditions (without shaking), $30^{\circ} \mathrm{C}$ in black, clearbottomed 96-well half area polystyrene plates with non-bonding surface (Corning \#3881) covered with Aluminium Thermowell Sealing Tape (Corning \#6570). The focal height was set to $4.9 \mathrm{~mm}$, and gain to 800 , with an excitation filter of $440-15 \mathrm{~nm}$ and emission filter of $480-15 \mathrm{~nm}$ and a dichroic cut-off of $460 \mathrm{~nm}$. Well measurements were taken by spiral average of $4 \mathrm{~mm}$ using the bottom optic, with 50 flashes per well and a cycle time of $1200 \mathrm{~s}$. The outer wells of the plate were not used. The experiments were performed in $100 \mu \mathrm{l}$ aliquots, in triplicate, each containing $100 \mu \mathrm{M}$ aS, $50 \mu \mathrm{M}$ ThT, $200 \mu \mathrm{M}$ DMPS, and $0.01 \%$ sodium azide in $20 \mathrm{mM}$ phosphate buffer $\mathrm{pH} 6.5$.

Lipid preparation for induced primary nucleation method

The mass of dry DMPS lipid powder was determined using an ultra-micro balance (Sartorius), and dissolved in $20 \mathrm{mM}$ sodium phosphate buffer $\mathrm{pH}$ 6.5 to a concentration of $2 \mathrm{mM}$. This was dissolved by shaking, in a $2 \mathrm{ml}$ Eppendorf tube, on a Thermomixer compact (Eppendorf), at $45^{\circ} \mathrm{C}$, 1400 r.p.m. for $3 \mathrm{~h}$. The solution was then freeze-thawed five times using dry ice and the thermomixer compact (Eppendorf) at $45^{\circ} \mathrm{C}$ and 500 r.p.m. The preparation of the vesicles was carried out by sonication, using a Soniprep 150 plus sonicator, set to an amplitude of 10.0, for five cycles of $30 \mathrm{~s}$ on and $30 \mathrm{~s}$ off.

\section{DLS measurements}

A sample of the vesicles produced at each step was diluted to $100 \mu \mathrm{M}$ in $20 \mathrm{mM}$ phosphate buffer $\mathrm{pH}$ 6.5. Their size distribution was measured by DLS, using a Zetasizer Nano ZSP (Malvern Instruments), to ensure a final consistent size of between 20 and $30 \mathrm{~nm}$ was obtained (Supplementary Fig. 2).

\section{Transmission electron microscopy}

aS samples from the end point of the aggregation kinetics were collected. Five microlitres of these samples were put onto glow-discharged Formvar/ carbon-coated, 200-mesh, copper grids for $1 \mathrm{~min}$. The samples were dried with a filter paper, and then washed twice with Milli-Q water for $1 \mathrm{~s}$, and removed each time with filter paper. The sample was stained by incubating the grids with $5 \mu \mathrm{l}$ Uranyl Acetate Zero (Agar Scientific) for $30 \mathrm{~s}$, followed by removal of the excess stain with a filter paper. The grids were left to air dry for $2 \mathrm{~h}$. The samples were imaged using a Transmission Electron Microscopy Jeol 2100 Plus (JEOL), operating at an accelerating voltage of $200 \mathrm{kV}$. Multiple grids were screened in order to obtain representative images of the samples.

\section{Reporting summary}

Further information on experimental design is available in the Nature Research Reporting Summary linked to this article.

\section{DATA AVAILABILITY}

The datasets generated during and/or analysed during the current study are available from the corresponding author on reasonable request.
Received: 13 March 2020; Accepted: 22 July 2020; Published online: 19 August 2020

\section{REFERENCES}

1. Meade, R. M., Fairlie, D. P. \& Mason, J. M. Alpha-synuclein structure and Parkinson's disease-lessons and emerging principles. Mol. Neurodegen. 14, 14 (2019).

2. Tuttle, M. D. et al. Solid-state NMR structure of a pathogenic fibril of full-length human alpha-synuclein. Nat. Struct. Mol. Biol. 23, 409-415 (2016).

3. Guerrero-Ferreira, R. et al. Cryo-EM structure of alpha-synuclein fibrils. elife $\mathbf{7}$. https://doi.org/10.7554/eLife.36402 (2018).

4. Li, Y. W. et al. Amyloid fibril structure of alpha-synuclein determined by cryoelectron microscopy. Cell Res. 28, 897-903 (2018).

5. Li, B. S. et al. Cryo-EM of full-length alpha-synuclein reveals fibril polymorphs with a common structural kernel. Nat. Commun. 9, 10 (2018).

6. Galvagnion, C. et al. Lipid dynamics and phase transition within alpha-synuclein amyloid fibrils. J. Phys. Chem. Lett. https://doi.org/10.1021/acs.jpclett.9b03005, 7872-7877 (2019).

7. Zhao, K. et al. Parkinson's disease associated mutation E46K of alpha-synuclein triggers the formation of a distinct fibril structure. Nat. Commun. 11, 2643 (2020).

8. Schweighauser, M. et al. Structures of alpha-synuclein filaments from multiple system atrophy. Nature. https://doi.org/10.1038/s41586-020-2317-6 (2020).

9. Guerrero-Ferreira, R. et al. Two new polymorphic structures of human full-length alpha-synuclein fibrils solved by cryo-electron microscopy. Elife 8. https://doi.org/ 10.7554/eLife.48907 (2019).

10. Stephens, A. D. et al. Extent of $\mathrm{N}$-terminus exposure of monomeric alphasynuclein determines its aggregation propensity. Nat. Commun. 11, 2820 (2020).

11. Viennet, T. et al. Structural insights from lipid-bilayer nanodiscs link alphasynuclein membrane-binding modes to amyloid fibril formation. Commun. Biol. 1, 12 (2018).

12. Boyer, D. R. et al. The alpha-synuclein hereditary mutation E46K unlocks a more stable, pathogenic fibril structure. Proc. Natl Acad. Sci. USA. https://doi.org/ 10.1073/pnas.1917914117 (2020).

13. Jao, C. C., Hegde, B. G., Chen, J., Haworth, I. S. \& Langen, R. Structure of membrane-bound alpha-synuclein from site-directed spin labeling and computational refinement. Proc. Natl Acad. Sci. USA 105, 19666-19671 (2008).

14. Madine, J., Doig, A. J. \& Middleton, D. A. A study of the regional effects of alphasynuclein on the organization and stability of phospholipid bilayers. Biochemistry 45, 5783-5792 (2006).

15. Galvagnion, C. et al. Lipid vesicles trigger alpha-synuclein aggregation by stimulating primary nucleation. Nat. Chem. Biol. 11, 229-U101 (2015).

16. Galvagnion, C. The Role of Lipids Interacting with alpha-synuclein in the pathogenesis of Parkinson's disease. J. Parkinsons Dis. 7, 433-450 (2017).

17. Agerschou, E. D. et al. An engineered monomer binding-protein for alpha-synuclein efficiently inhibits the proliferation of amyloid fibrils. Elife 8, 31 (2019).

18. Strohaker, T. et al. Structural heterogeneity of alpha-synuclein fibrils amplified from patient brain extracts. Nat. Commun. 10, 12 (2019).

19. Boyer, D. R. et al. Structures of fibrils formed by alpha-synuclein hereditary disease mutant $\mathrm{H} 50 \mathrm{Q}$ reveal new polymorphs. Nat. Struct. Mol. Biol. 26, 1044-1052 (2019).

20. Fanning, S., Selkoe, D. \& Dettmer, U. Parkinson's disease: proteinopathy or lipidopathy? NPJ Parkinsons Dis. 6, 3 (2020).

21. Rawat, A., Langen, R. \& Varkey, J. Membranes as modulators of amyloid protein misfolding and target of toxicity. Biochimica Et. Biophysica ActaBiomembranes 1860, 1863-1875. https://doi.org/10.1016/j.bbamem.2018.04.011 (2018).

22. Varkey, J. et al. Membrane curvature induction and tubulation are common features of synucleins and apolipoproteins. J. Biol. Chem. 285, 32486-32493 (2010).

23. Perni, M. et al. Multistep inhibition of alpha-synuclein aggregation and toxicity in vitro and in vivo by trodusquemine. ACS Chem. Biol. 13, 2308-2319 (2018).

24. Buell, A. K. et al. Solution conditions determine the relative importance of nucleation and growth processes in alpha-synuclein aggregation. Proc. Natl Acad. Sci. USA 111, 7671-7676 (2014).

25. Brown, J. W. P. et al. Kinetic barriers to alpha-synuclein protofilament formation and conversion into mature fibrils. Chem. Commun. 54, 7854-7857 (2018).

26. Flagmeier, P. et al. Mutations associated with familial Parkinson's disease alter the initiation and amplification steps of alpha-synuclein aggregation. Proc. Natl Acad. Sci. USA 113, 10328-10333 (2016). 
27. Ross, B. M., Mamalias, N., Moszczynska, A., Rajput, A. H. \& Kish, S. J. Elevated activity of phospholipid biosynthetic enzymes in substantia nigra of patients with Parkinson's disease. Neuroscience 102, 899-904 (2001).

28. Dettmer, U. Rationally designed variants of alpha-synuclein illuminate its in vivo structural properties in health and disease. Front. Neurosci. 12, 14 (2018).

29. Bendor, J. T., Logan, T. P. \& Edwards, R. H. The function of alpha-synuclein. Neuron 79, 1044-1066 (2013)

30. Fusco, G. et al. Structural basis of membrane disruption and cellular toxicity by alpha-synuclein oligomers. Science 358, 1440-+ (2017).

31. Fusco, G. et al. Direct observation of the three regions in alpha-synuclein that determine its membrane-bound behaviour. Nat. Commun. 5, 8 (2014).

32. Pujols, J. et al. High-throughput screening methodology to identify alphasynuclein aggregation inhibitors. Int. J. Mol. Sci. 18. https://doi.org/10.3390/ ijms18030478 (2017).

33. Volles, M. J. \& Lansbury, P. T. Jr. Relationships between the sequence of alphasynuclein and its membrane affinity, fibrillization propensity, and yeast toxicity. J. Mol. Biol. 366, 1510-1522 (2007).

\section{ACKNOWLEDGEMENTS}

J.M.M., R.J.W. and R.M.M. thank BRACE for award of a Ph.D. studentship (BR16/064). This work is also supported by a project grant and an equipment grant from Alzheimer's Research UK (ARUK-PG2018-003; ARUK-EG2018A-008). We also thank Philip Fletcher, Diana Lednitzky and Silvia Martinez Micol for their assistance with the transmission electron microscope.

\section{AUTHOR CONTRIBUTIONS}

J.M.M. directed the research. R.M.M. conducted the experiments, and expressed, purified and characterised the protein. R.M.M., R.J.W. and J.M.M. participated in experimental design, analysis of the data and writing the paper.

\section{COMPETING INTERESTS}

J.M.M. is an advisor to Sapience Therapeutics. The authors declare no other competing interests.

\section{ADDITIONAL INFORMATION}

Supplementary information is available for this paper at https://doi.org/10.1038/ s41531-020-00122-1.

Correspondence and requests for materials should be addressed to J.M.M.

Reprints and permission information is available at http://www.nature.com/ reprints

Publisher's note Springer Nature remains neutral with regard to jurisdictional claims in published maps and institutional affiliations.
Open Access This article is licensed under a Creative Commons Attribution 4.0 International License, which permits use, sharing, adaptation, distribution and reproduction in any medium or format, as long as you give appropriate credit to the original author(s) and the source, provide a link to the Creative Commons license, and indicate if changes were made. The images or other third party material in this article are included in the article's Creative Commons license, unless indicated otherwise in a credit line to the material. If material is not included in the article's Creative Commons license and your intended use is not permitted by statutory regulation or exceeds the permitted use, you will need to obtain permission directly from the copyright holder. To view a copy of this license, visit http://creativecommons. org/licenses/by/4.0/.

(c) The Author(s) 2020 